\title{
Ancora qualche considerazione sul problema inverso della geoelettrica
}

\author{
A. BEILIIII
}

Ricevuto il 31 Ottobre 1966

\begin{abstract}
Rassunto. - LiA. mostra l'indirizzo seguito nell'interpretazione dei rilevamenti geoelettrici, a corrente stazionaria, avvalendosi delle solnzioni del "problema inverso" geoelettrico.

Si completa il metodo dato nel 1959 ed esposto (i) in " Zeitschrift für Geophysik" - Ileft $3-(113-1+2)$.
\end{abstract}

Summarx. - The problem of deducing from electrical potentials observed at the surface (horizontally uniform Earth) the unknown variation of the conductivity with depth is developed, and the anisotropic case is discussed.

Zusamenfassuvg. - Der V. behandelt das "umgekehrte gesel. Problem " und folgt dem von Langer gegebenen strengen analytischen Ansatz; entwickelt der V. einen nenen Algorithmüs, u.s.v.w.

Da Langer a Slichter a Tikhonov, all' $\mathrm{A}$. ( $\left.{ }^{1}\right)$, come ricorda esplicitamente A. L. Cheter (richiamando i motivi delle trattazioni, Are. Sc. ITRSS), il terreno elettricamente conduttore, convogliante corrente stazionaria (elettrotelluriea artificiale o naturale), viene assunto quale un semispazio infinito, uniforme, piano-orizzontale, con conduttività geoelettrica dipendente solo dalla profondità $\sigma(z)$, a partire natumalmente dalla superficie piana del suolo.

Basta considerare inoltre, applicato in superficie, un elettrodo puntiforme, un reoforo che immetta ad es. cece (corrente continua). 
Anche la non infrequente elettroanisotropia potrà considerarsi variabile solo con la profondità, a conduttività, in ogni piano orizzontale, indipendente dalla direzione. Si arramno sempre condizioni simmotriche rispetto allasse verticale passante per leelettrodo superficiale cilindrico verticale o simmetrico-rerticale (sferico, emisferico, ellissoidico, ronicos).

I geopotenziali elettrici, rilevabili con lines equipotenziali o di corrente, alla superficie del terreno, su vaste aree intorno alleceltrodo d'emergizzazione, doverbbero permettere (finalità della prospezione) di risalire da questi valori di misurazione all'incognita funzione di conduttività $\sigma(z)$, a ciò costituisce il cosidetto "problema inverso geoelettriceo ".

A questo problema, com'è stato più volte autorevolmente rilevato, è stata rivolta sempre scarsa attenzione, mentre per il problema inverso - epperò diretto — abbondano notoriamente studi, procedimenti risolutivi, interpretativi, spesso grossolani a a carattere del tutto empirico $\left(^{2}\right)$ :

Comunque il cosidetto problemal inverso geoelettrico consiste nel determinate la " $\sigma(z) "$ esistente nel mezzo terroso, supposto note: $\sigma_{o}=\sigma(o)$, e la distribuzione $V(r)$ del geopotenziale sulla superficie, in funzione della distanza "r" dill'elettrodo di potenza, granderze tutte accertabili com alta preceisione.

Il punto più rigoroso di partenzı è dato, com’è noto, dalla trattazione del Langer $\left(^{1}\right)$, condotta a più riphese nel "Bulletin of American Mathematical Society".

Il problema cosidetto inverso (di per sé diretto), di dedure cioé dai potenziali elettrei rilevati alla superficie del suolo, l’incognite conduttivita elettriche, variabili con la profondità del tereno, si ridues ad un problema di valore al contorno, di tipo non comume, in quanto la funzione da trovare esprine la variazione incognita da un punto all'altro di costante fisica del materiale (la conduttività), mentre la funzione stessa si considema nota ai limiti.

Il problema poi inverso a questo, detto impropriamente diretto, a cui si è più abituati, assumendo inveree nota (per tentativi) la precedente incognita funzione conduttività, e risolvente la questione relativa al campo potenziale in superficie prericostruibile, è più semplice, porta a soluzioni con valori di confronlo rispetto a quelli determinati ad es. dalle misure potenziometriche, modificando o adattando i presupposti se il confronto fallisce funo ad ottenere una sufficiente concordanza. Procedimento pereiò per tentativi, essenzialmente empirico per le irreali schematizzazioni, nonostante il grande sucessso incontrato in pratica, più che altro propagandistico, oggi in declino. 
Denotiamo ora con $K(\lambda)$ la funzione "nucleo ":

$$
K(\lambda)=\frac{2 \pi \sigma_{o}}{c} \lambda \int_{0}^{\infty} V(r) J_{0}(\lambda r) r d r \text { (c intensità di corrente) }
$$

per cui note $\sigma_{o}, V(r), K\left(\lambda_{)}\right)$si può considerare una funzione nota di $\lambda$.

Assumendo un elettrodo di raggio $a$, invece che puntiforme, si dovrà moltiplicare tale funzione $K(\lambda)$ per il rapporto:

$$
(\lambda a / \operatorname{sen} \lambda a) \text {. }
$$

Come già è apparso nel lavoro del Langer e nel nostro: "Berechnung der elektrischen Leitfähigkeit u.s.v." (1), il problema si può affrontare nei seguenti termini.

Consideriamo l'equazione differenziale:

$$
\frac{d^{2} Z}{d z^{2}}+\frac{\sigma^{\prime}(z)}{\sigma(z)} \frac{d Z}{d z} \quad 22 Z=\text {. }
$$

la quale ammette una soluzione (determinata a meno di un fattore costante): $Z(z, \lambda)$, che si annulla per $z \rightarrow \infty$. Questa soluzione verri in seguito indicata con $Z_{1}(z, \lambda)$, là ove ci sarà bisogno di distinguerla da un'altra linearmente indipendente come $Z_{2}(z, \lambda)$.

Si dovrà avere cioé per $Z_{1}$ :

$$
Z_{1}(\infty, \lambda)=0 \text {. }
$$

Mediante tale $Z(z, \lambda)$, la funzione $K(\lambda)$ potrà essere espressa da:

$$
K(\lambda)=-\lambda Z(0, \lambda) / Z^{\prime}(0, \lambda)
$$

dove l'apice indica la derivazione prima rispetto alla variabile $z$.

Potendo convenire di normalizzare le $Z$ (nelle $Z$ è disponibile ancora un fattore), si pone la condizione ulteriore:

$$
Z^{\prime}(0, \lambda)=-\lambda
$$

nel qual caso la [2] diventa semplicemente:

$$
K(\lambda)=Z(0, \lambda) .
$$

Il problema consiste nel determinare il rapporto:

$$
\sigma^{\prime}(z) / \sigma(z)
$$

in maniera tale che la soluzione del sistema [1] e [1'] soddisfi anche la relazione [2] (l'apice in alto a destra della $\sigma$, è indice di derivazione). 
Il Langer risolse questo problema (cosi formulato), nell'ipotesi che:

1) la funzione $K(\hat{\lambda})$ ammetta umo sviluppo in potenze inverse di $\lambda$,

2) il rapporto $\left(\sigma^{\prime} / \sigma\right)$ sia una funzione analitica di $r$ (almeno in prossimità di $z=0$ ).

Gli svantaggi che presenta il metodo originale del Langer sono stati da noi ampiamente discussi e rimossi in [2]. Causa tali svantaggi L. B. Slichter si limitò prima ad esporre casistiche $\left({ }^{1}\right)$, in cui l'equazione [1] ammette soluzioni in forma semplice.

I) agni posizione assunta si ricava immediatamente però tutta una "famiglia di conduttività " per cui il problema è risolubile semiempiricamente, e non del tutto generalmente, nella seguente maniera: supposta nota la funzione $Z(z, \lambda)$, in corrispondenza ad una certa conduttività $\sigma(z)$, si trae dalle [1] e [1'], che alla $\sigma(z+c)$ corrisponderà la $Z(z+c, \lambda)$; il corrispondente valore $K(\lambda)$ sarà pertanto dato per la [2] da:

$$
K(\lambda)=-\frac{\lambda Z(c, \lambda)}{Z^{\prime}(c, \lambda)}
$$

In secondo luogo si può rilevare che da ogni famiglia di curve è ottenibile la famiglia inversa, è possibile cioè dimostrare che se:

$$
\sigma_{1}(z) \cdot \sigma_{2}(z)=\mathrm{cost},
$$

anche il prodotto delle due funzioni $K$ :

$$
K_{1}(\lambda) K_{2}(\lambda)=1 \text {. }
$$

Infatti la [4] implica (derivando $\log \sigma_{1}+\log \sigma_{2}=$ cost) che:

$$
\frac{\sigma_{1}^{\prime}}{\sigma_{1}}+\frac{\sigma_{2}^{\prime}}{\sigma_{2}}=\mathbf{0}
$$

La [1] d'altra parte dà:

$$
\frac{d^{2} Z^{(1)}}{d z^{2}}+\frac{\sigma_{1}}{\sigma_{1}} \frac{d Z^{(1)}}{d z}+\lambda^{2} Z^{(1)}=0 ; \frac{d^{2} Z^{(2)}}{d z^{2}} ; \frac{\sigma_{2}^{\prime}}{\sigma_{2}} \frac{d Z^{(2)}}{d z}-\lambda^{2} Z^{(2)}=0 .
$$

Moltiplicando la prima delle [7] per $\frac{d Z^{(2)}}{a z}$, la seconda per $\frac{d Z^{(1)}}{d z}$, quindi sommando e integrando tra $(0$ e $\infty)$, tenendo conto della [6], si ottiene:

$$
\int_{0}^{\infty}\left(\frac{d^{2} Z^{(1)}}{d z^{2}} \frac{d Z^{(1)}}{d z}+\frac{d^{2} Z^{(2)}}{d z^{2}} \frac{d Z^{(2)}}{d z}\right) d z-\lambda^{2} \int_{0}^{\infty}\left(\frac{d Z^{(2)}}{d z} Z^{(1)}+\frac{d Z^{(1)}}{d z} Z^{(2)}\right) \mid d z=0,
$$


ovvero:

$$
\left[\left.\frac{d Z^{(1)}}{d z} \cdot \frac{d Z^{(2)}}{d z}\right|_{0} ^{\infty}-\lambda^{2}\left|Z^{(1)} Z^{(2)}\right|_{0}^{\infty}=0\right.
$$

da cui tenendo presenta la $\left[1^{\prime}\right]$ :

$$
Z^{(1)^{\prime}}(0, \lambda) Z^{(2)^{\prime}}(0, \lambda)=\lambda^{2} Z^{(1)}(0, \lambda) Z^{(2)}(0, \lambda),
$$

la quale, in base alla definizione di $K_{1}(\lambda)$ e $K_{2}(\lambda)$, coincide con la [5]. In questa maniera si può ottenere — da ogni famiglia di conduttivitàanche quella inversa, e la casistica ovviamente possiamo estenderla, coprendo una quantità di casi possibili nella prospezione geoelettrica.

I) Esaminiamo pertanto le funzioni $Z$ che coinvolgono quelle di Bessel (le notazioni per le funzioni di Bessel sono quelle adottate da Watson). Con il cambiamento di variabile:

$$
Z=\sigma^{-1 / 2 \cdot \varkappa}
$$

lat [1] si trasforma in:

$$
\frac{d^{2} u}{d z^{2}}-\left[1+\frac{1}{4 \lambda^{2}}\left\{2\left(\frac{\sigma^{\prime}}{\sigma}\right)+\left(\frac{\sigma^{\prime}}{\sigma}\right)^{2}\right\}\right] u=0,
$$

un'equazione che ammette soluzioni che si esprimono mediante funzioni di Bessel: introducendo il parametro Besseliano $v$, si ha:

$$
2\left(\frac{\sigma^{\prime}}{\sigma}\right)+\left(\frac{\sigma^{\prime}}{\sigma}\right)^{2}=\frac{\left(4 v^{2}-1\right)}{Z} .
$$

Tale equazione allora ha come soluzione grenerale (il che si può verificare immediatamente per sostituzione):

$$
\sigma=B e^{\left(1-2 \eta^{\prime \prime}\right)} \cdot\left(1+A Z^{2 \nu}\right)^{2} .
$$

In questa la soluzione della [9], che soddisfa la condizione all'infinito, è dat à dalla:

$$
\begin{gathered}
u=z^{1 / 2} \cdot K_{v},(\lambda z), \text { da cui si ottiene per la } Z=u \cdot \sigma^{-1 / 2} ; \\
Z_{1}(z, \lambda)=c\left|\frac{Z^{v} K v(\lambda z)}{1+A Z^{2 v}}\right| .
\end{gathered}
$$

Si ha quindi ehe per una conduttiviti del tipo:

$$
\sigma=B(z+c)^{1-2 \nu},\left[1+A(z+c)^{2}{ }^{\prime}\right],
$$


la $K(\lambda)$ è data dalla formula:

$$
[K(\lambda)]^{-1}=\frac{K_{\nu-1}\left(\lambda_{c} c\right)}{K_{v}(\lambda c)}+\frac{2 \nu}{\lambda_{c}} \cdot \frac{A e^{2 \nu}}{1+A c^{2 \nu}},
$$

che deriva appunto sostituendo la [11] nella [3] (non riportiamo i grafici relativi, per brevità e concisione).

Si osservi che nelle [12] e [13] sono disponibili 3 parametri, i quali si possono abbastanza bene adattare per adeguarsi alle curve sperimentali (speciali nomogrammi permettono la variazione relativa di tali parametri).

Si dispone così di un certo numero di curve per $v$ semiinteri, nel qual caso le $K_{v}$ sono esprimibili mediante funzioni elementari (esponenziali e polinomi in $\lambda^{-1}$ ).

II) Caso funzione esponenziale: esso è il più elementare e già esaminato; infatti per $\sigma$ della forma $\sigma=\sigma_{0} e^{2 b z}$, l'equazione [1] passa a coefficienti costanti e si scrive: $z^{\prime \prime}+2 b z^{\prime}-\lambda^{2}=0$, la cui soluzione che si annulla all'infinito, è data da:

$$
Z=\exp \left\{-b\left[1+\sqrt{1+(\lambda / b)^{2}}\right]\right\}
$$

Pertanto si ha per $K(\lambda)$ :

$$
\Pi(\lambda)=\frac{b}{\lambda}[\sqrt{1}+(\lambda / b)-1]
$$

III) Caso di funzioni iperboliche (integra i precedenti).

Qualora la conduttività $\sigma$ assuma la forma:
a) $\sigma=A c h^{2} \omega(z+c)$
b) $\sigma=A \operatorname{sh}^{2} \omega(z+c)$,

saranno anche note le soluzioni della [1]. Quelle che soddisfano la [1] sono date rispettivamente dalle:
a) $Z=\exp \frac{\left[-z \sqrt{\lambda^{2}+\omega^{2}}\right]}{\operatorname{sh} \dot{\omega}(z+c)}$
b) $Z=\exp \frac{\left[-z \sqrt{\lambda^{2}+\omega^{2}}\right]}{\operatorname{ch} \dot{\omega}(z+c)}$

da cui si ricava per le funzioni $K(\lambda)$ :
a) $\quad K(\lambda)=\frac{\lambda}{\left(\sqrt{\lambda^{2}+\omega^{2}}+\dot{\omega} \operatorname{ch} \omega c\right)}$
b) $K(\lambda)=\frac{\lambda}{\left(\sqrt{\lambda^{2}+\omega^{2}}+\dot{\omega} \operatorname{th} \omega c\right)}$. 
Le posizioni I, II, III danno molteplici e varie curve per $K\left(\lambda_{)}\right)$, per cui è possibile determinare direttamente le corrispondenti $\sigma$. In più, come è stato suesposto, si possono aggiungere tutte le curve inverse derivabili (casistiche analitiche e grafiche del genere aiutano in modo razionale l'interpretazione).

I casi I, II, III, esauriscono quelli semplici, un breve elenco che si può però incrementare, come abbiamo mostrato altrove ( $\left.{ }^{1}\right)$.

Una particolare utile estensione, è per il "terreno stratificato », in ogni strato del quale vale una formula sufficientemente semplice per $\sigma^{\prime} / \sigma$. Nel passaggio da uno strato all'altro valgono allora le condizioni di continuità date dalle:

$$
Z_{i}=Z_{i+1}, \quad \sigma_{i} Z_{i}=\sigma_{i+1} Z_{i+1} .
$$

Riferiamoci a questo punto ad un terreno composto da $n$ strati orizzontali indefiniti sovrapposti, e si indichi con $\sigma_{i}(z)$ la funzione rappresentante la conduttività dell'iesimo strato, con $\sigma_{n+1}(z)$ indicante quella del suolo sottostante (basale); sia inoltre $h_{2}$ la profondità a cui perviene l'iesimo strato (profondità dell'imbasamento).

Indichiamo le soluzioni della [1], per l'iesimo strato con:

$$
x_{i}=Z_{i, 1}(z, \lambda), \quad y_{i}=Z_{i, 2}(z, \lambda) .
$$

In base alla nostra convenzione, $x_{i}$ rappresenta quella soluzione che per $Z \rightarrow \infty$ si annulla.

La soluzione relativa all'iesimo strato sarà quindi rappresentabile da:

$$
Z_{i}=A_{i} x_{i}+B_{i} y_{i} .
$$

mentre nel suolo sottostante si avrà (per la condizione all' $\infty$ ):

$$
Z_{n+1}=A_{n+1} \cdot x_{n+1} \text {. }
$$

Puó convenire impiegare la $Z_{1}$ normalizzata, come abbiamo posto nella [1"], e si ottiene:

$$
A_{1} x_{1}^{\prime}(0)+B_{1} y_{1}^{\prime}(0)=-\lambda .
$$

Le condizioni di continuità risultano:

$$
\begin{aligned}
& A_{i} x_{i}\left(h_{i}\right)+B_{i} y_{i}\left(h_{i}\right)-A_{i+1} x_{i+1}\left(h_{i}\right)-B_{i+1} y_{i+1}\left(h_{i}\right)=0, \\
& \begin{array}{l}
A_{i} \sigma_{i}\left(h_{i}\right) x_{i}^{\prime}\left(h_{i}\right)+B_{i} \sigma_{i}\left(h_{i}\right) y_{i}^{\prime}\left(h_{i}\right)-A_{i+1} \sigma_{i+1}\left(h_{i}\right) x_{i+1}\left(h_{i}\right)- \\
\quad-B_{i+1} \sigma_{i+1}\left(h_{\imath}\right) y_{i+1}^{\prime}\left(h_{i}\right)=0 . \\
(i=1,2, \ldots n-1)
\end{array}
\end{aligned}
$$


$A_{n} x_{n}\left(h_{n}\right)+B_{n} y_{n}\left(h_{n}\right)-A_{n+1} x_{n+1}\left(h_{n}\right)=0$
$A_{n} \sigma_{n}\left(h_{n}\right) x_{n}^{\prime}\left(h_{n}\right)+B_{n} \sigma_{n}\left(h_{n}\right) y_{n}^{\prime}\left(h_{n}\right)-A_{n+1} \sigma_{n+1}\left(h_{n}\right) x_{n+1}\left(h_{n}\right)=0$.

Le [15], [16] e [16'], formano un sistema di $(2 n+1)$ equazioni in $(2 n+1)$ incognite: $A_{i}(\operatorname{con} i=1, \ldots n+1)$ e $B_{i}(\operatorname{con} i=1 \ldots n)$.

Determinate le $A_{i}, B_{i}$, da questo sistema si ottiene per la $K(\lambda)$ (v. [3]):

$$
K(\hat{\lambda})=A_{1} x_{1}(0)+B_{1} y_{1}(0)
$$

Limitandoci ad un solo strato $(n=1)$, posto:

$$
h_{1}=h, \quad \frac{\sigma_{i+1}(h)}{\sigma_{i}(h)}=r_{i}
$$

risolvendo il sistema delle $t$ equazioni ed applicando la [17], si perviene, alla:

$$
K(\lambda)=-\lambda \frac{C x_{1}(0)-D y_{1}(0)}{C x_{1}^{\prime}(0)-D y_{1}^{\prime}(0)}
$$

con le funzioni:

$$
C^{\prime}=x_{2}(h) y_{1}^{\prime}(h)-r y_{1}(h) y_{2}^{\prime}(h): \quad D=-x_{2}(h) x_{1}^{\prime}(h)-r x_{1}(h) x_{2}^{\prime}(h) .
$$

Lat [19] si può applicare successivamente ad una serie numerosa di casi particolari (tutti questi casi, appariranno, con i precedenti grafici, in apposito Volume).

$\alpha)$

$$
\sigma_{1}=\sigma(z+c), \quad z<h ; \quad \sigma_{2}=\sigma(h+c), \quad z>0 .
$$

La linearità rientra nell'assunzione (I), con $A=0$ e $y=0$, per cui si ha:

$$
x_{1}=K_{0}[\lambda(z+c)], \quad y_{1}=I_{0}[\lambda(z+c)],
$$

mentre ovviamente; essendo $\sigma_{2}$ costante, si ottiene (caso II: $b=(0)$ :

$$
x_{2}-\lambda z
$$

Inoltre essendo $\sigma(z)$ funzione continua in $x=h$, si ha:

$$
r=1 \text {. }
$$

Tenendo conto che: $K_{0}^{\prime}=-K_{1}, I_{1}^{\prime}=I_{1}$, la [19] diventa:

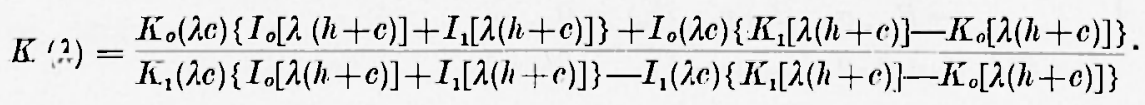




$$
\sigma_{1}=c(z+c), \quad z<h ; \quad \sigma_{2}=\infty, \quad z>h,
$$

(questo caso differisce dal precedente in quanto ora $r=\infty$ ) e analogamente si ottiene:

$$
K(\lambda)=\frac{K_{o}(\lambda c) I_{o}[\lambda(h+c)]-I_{o}(\lambda c) K_{o}[\lambda(h+c)]}{K_{1}(\lambda c) I_{o}[\lambda(h+c)]+I_{1}(\lambda c) K_{o}[\lambda(h+c)]} .
$$

$\gamma)$

$$
\sigma_{1}=\sigma(z+c), \quad z<h ; \quad \sigma_{2}-0, \quad z>0 .
$$

Qui differiamo dal precedente assunto solo per il fatto che ora $r=0$; dalla [19] otteniamo allora:

$$
K(\hat{\lambda})=\frac{K_{o}(\lambda c) I_{1}\left[\lambda(h+c)+I_{o}(\lambda c) K_{1}[\lambda(h+c)]\right.}{K_{1}(\lambda c) I_{1}\left[\lambda(h+c)-I_{1}(\lambda c) K_{1}[\lambda(h+c)]\right.} .
$$

反)

$$
\sigma_{1}=a_{1}\left(z+c_{1}\right), \quad z<h ; \quad \sigma_{2}=\alpha_{2}\left(z+c_{2}\right), \quad z>0,
$$

con:

$$
a_{1}\left(h+c_{1}\right)=a_{2}\left(h+c_{2}\right) .
$$

Anzitutto dalla condizione sopraseritta consegue che $r=1 ; x_{1}$ e $y_{1}$ hanno la stessa espressione dei casi precedenti $\left(c=c_{1}\right)$, mentre evidentemente $x_{1}=K_{o}\left[\lambda\left(z+c_{2}\right)\right]$.

Sostituendo questa espressione nella [19], perveniamo alla:

$K(\lambda)=\frac{K_{o}\left(b_{o}\right)\left[I_{1}\left(b_{1}\right) K_{o}\left(b_{2}\right)+I_{o}\left(b_{1}\right) K_{1}\left(b_{2}\right)\right]+I_{o}\left(b_{2}\right)\left[K_{o}\left(b_{2}\right) K_{1}\left(b_{1}\right)-K_{o}\left(b_{1}\right) K_{1}\left(b_{2}\right)\right]}{K_{1}\left(b_{o}\right)\left[I_{1}\left(b_{1}\right) K_{o}\left(b_{2}\right)+I_{o}\left(b_{1}\right) K_{1}\left(b_{2}\right)\right]-I_{1}\left(b_{o}\right)\left[K_{o}\left(b_{2}\right) K_{1}\left(b_{1}\right)-K_{o}\left(b_{1}\right) K_{1}\left(b_{2}\right)\right],}$ con:

$$
b_{o}=\lambda c_{1} ; \quad b_{1}=\lambda\left(h+c_{1}\right) ; \quad b_{2}=\lambda\left(h+c_{2}\right) .
$$

Nella situazione di "strati omogenei ", il sistema [15] e [16] si semplifien: tenendo conto che,

lia

$$
\begin{gathered}
x_{1}-\hat{\imath}^{2} z, \quad y_{1}=e^{\hat{\lambda} z} \\
K(\lambda)=\left(A_{1}+B_{1}\right),
\end{gathered}
$$

assume la forma:

$$
K(\lambda)=\left(1+2 B_{1}\right)
$$

ricorrendo alla [15] (che ora diviene $A_{1}-B_{1}=1$ ): basterà quindi calcolare la $B_{1}$.

Per il numero di strati $n=2$ si ritrovano le formule esplicite e ben note di Inummel-Stefanescu-Ollendorf (di difficile applicazione per $n>2$ ). 
Questo metodo, come s'è visto brevemente, consiste nel determinare la $K(\lambda)$ dai dati sperimentali, e di confrontarla con qualcuna delle curve (v. Albcir) previamente calcolate, o agevolmente adattabili.

E immediata pure l'esemplificazione per tre conduttività diverse, anche se non differiscono molto tra di loro, o per più di tre, per illustrare l'influenza della conduttività sull'andamento della $K(\lambda)$ e della $V(r)$.

Riferiamoci infine ad un suolo in eui la conduttività, oltre a dipendere dalla sola profondità $Z$, presenta unicamente simmetria intorno all'asse $z$. In altre parole l'ellissoide di conduttività ha un asse verticale, e gli altri due uguali tra di loro.

Indichiamo allora con $\sigma_{z}(z)$ la conduttività verticale,e con $\sigma_{\varrho}(z)$ la conduttività orizzontale, il "rapporto di anisotropia " risulterà:

$$
\left.o(z)=\sqrt{\frac{\sigma_{\mathrm{t}}(z)}{\sigma_{z}(z)}}, \text { ("indice anisotropico } "\right) \text {. }
$$

Il problema della determinazione del "potenziale " si riduce qui al Iitrovamento d'una funzione $\varphi(z, \varrho)(\varrho=$ distanza dall'asse $z)$, che soddisfa alla seguente equazione differenziale:

$$
\frac{\partial^{2} \varphi}{\partial z^{2}}+\frac{1}{\sigma_{z}} \cdot \frac{d \sigma_{z}}{d z} \quad \frac{\partial \varphi}{\partial z}-\tilde{q}^{2}\left(\frac{\partial^{2} \varphi}{\partial \varrho^{2}}+\frac{1}{\varrho} \frac{\partial \varphi}{\partial \varrho}\right)=0
$$

ron le seguenti condizioni al contorno:

$$
\left\{\begin{array}{l}
z=0, \quad \frac{\partial \varphi}{\partial z}=0, \quad \varphi \simeq-\frac{c}{2 \pi \varrho \sqrt{\sigma_{\varrho}(0) \sigma_{z}(0)}}, \quad \text { per } \varrho \rightarrow 0 \\
z \rightarrow \infty, \text { oppure } \varrho \rightarrow \infty, \quad \varphi \rightarrow 0 .
\end{array}\right.
$$

Si dimostra, è ben noto, che con un cambiamento di variabile $z$ in una nuova variabile $w$, il problema si trasforma in quello isotropico:

$$
\frac{\partial^{2} \varphi}{\partial w^{2}}+\frac{1}{u} \frac{d u}{d w} \frac{\partial \varphi}{\partial w}+\frac{\partial-\varphi}{\partial \varrho^{2}}+\frac{1}{\varrho} \frac{\partial \varphi}{\partial \varrho}=0
$$

dove:

$$
\left\{\begin{array}{l}
w=0, \quad \frac{\partial \varphi}{\partial w}=0, \quad \varphi \simeq-\frac{c}{\varrho \cdot 2 \pi w(0)}, \text { per } \varrho \rightarrow 0 \\
w=\infty, \text { oppure } \varrho-0, \quad \varphi=0 .
\end{array}\right.
$$

Determineremo ora le nuove variabili $w(z)$, e $u(w)$ (conduttività equivalente) che trasformano l'equazione iniziale in queste ultime. 
Si ha infatti:

$$
\frac{\partial \varphi}{\partial z}-\frac{\partial \varphi}{\partial w} \cdot \frac{d w}{d z} ; \frac{\partial^{2} \varphi}{\partial z^{2}}-\frac{\partial^{2} \varphi}{\partial w^{2}}\left(\frac{d w}{d z}\right)^{2}+\frac{\partial \varphi}{\partial w} \frac{d^{2} w}{d z^{2}} .
$$

Sostituendo questi valori nell'equazione di partenza [20] e dividendo per $\left(\frac{d w}{d z}\right)^{2}$, si ottiene:

$$
\frac{\partial^{2} \varphi}{\partial w^{2}}+\left[\frac{\frac{d^{2} w}{d z^{2}}}{\left(\frac{d w}{d z}\right)^{2}}+\frac{1}{\sigma_{z}} \frac{d \sigma_{z}}{d w}\right] \frac{\partial \varphi}{\partial w}+\frac{q^{2}}{\left(\frac{d w}{d z}\right)^{2}}\left|\frac{\partial^{2} \varphi}{\partial \varrho^{2}}+\frac{1}{\varrho} \frac{\partial \varphi}{\partial \varrho}\right|=0
$$

Per far coincidere la [22] con la [20], è sufficiente che si abbia:

$$
\begin{gathered}
q=\frac{d w}{d z}, \\
\left\lfloor\frac{d^{2} w}{\left.\frac{d z^{2}}{\left(\frac{d w}{d z}\right)^{2}}\right\rceil+\frac{1}{\sigma_{z}} \frac{d \sigma_{z}}{d w}=\frac{1}{u} \frac{d u}{d w},}\right.
\end{gathered}
$$

e inoltre per far coincidere la [21] con la [21'] basta richiedere:

$$
\begin{gathered}
w=0 \quad \text { per } z=0 \\
w(0)=\sqrt{\sigma_{z}(0) \sigma_{\varrho}(0)}=\sigma_{z}(0) q(0) .
\end{gathered}
$$

Dalla [23] e [25] si trae immediatamente:

$$
w(z)=\int_{0}^{z} q(z) d z .
$$

Inoltre dalla [23] si ha:

che insieme con la:

$$
\left\lceil\frac{\frac{d^{2} w}{d z^{2}}}{\left(\frac{d w}{d z}\right)^{2}}\right\rceil=\frac{\frac{d q}{d z}}{q^{2}}=q^{-2} \frac{d q}{d z}
$$

$$
\frac{d q}{d z}-\frac{d q}{d w} \cdot \frac{d w}{d z}-\frac{d q}{d w} \cdot q
$$

dà:

$$
\frac{\frac{d^{2} w}{d z^{2}}}{\left(\frac{d w}{d z}\right)^{2}}=q^{-1} \frac{d}{d w}
$$


Pertanto la [25] si puó scrivere:

$$
q^{-1} \frac{d q}{d w}+\sigma_{2}^{-1} \frac{d \sigma_{n}}{d w}=w^{-} \cdot \frac{d u}{d w}
$$

la quale integrata dà:

$$
\lg q+\lg \sigma_{2}==\lg u+\cos f, \quad \text { oppure } w=A q \sigma_{z} .
$$

Dil quest'ultima, tenuto conto della [26], si ottiene $A=0$ e quindi:

$$
u=q \sigma_{z}=\sqrt{\sigma_{\varrho} \sigma_{z}}
$$

Si ha dunque, come risultato, che per risolvere il problemat anisotropico basta, invece della profondità, introdurre una nuova variabile:

$$
w=\int_{0}^{z} \varrho(z) d z,
$$

e risolvendo il problema con una "condutlività isotropica " $u$ :

$$
u=q \sigma_{z}=\sqrt{\sigma_{0} \sigma_{z}} .
$$

Si vede che il problema inverso non è uniromamente determinabile, potendosi solo ricavare la $u=\sqrt{\sigma_{Q} \sigma_{z}}$, in funzione di $w$.

Sarebbe necessatria, pereiò, qualche ulteriore conoseenzal per ricavare la $w$ in funzione di $z$, e, di conseguenzal la $q=\frac{d w}{d z}$.

Considerando infatti la $q$ come una funzione nota di $\sigma_{z}$, allora la relazione:

$$
u(w)=f\left(\sigma_{z}\right) \cdot \sigma_{z},
$$

permette - essendo nota la $u(w)$ - di determinare la $\sigma_{z}$ in funzione di $w$, e di consegruenzil anche $q=\frac{d w}{d z}$.

Ponendo la funzione $q=F^{\prime}(w)$, mercè la [23] si puó determinare $Z$ in funzione di $w$. Si ha infatti:

$$
Z=\int_{0}^{w} \frac{d w}{F(w)},
$$

da cui poi si ricava $w$ in funzione di $z$, c.ll.t. 


\section{BIBLIOGRAFIA}

(1) Langer R. E., An inverse Problem in Differential Equation. "Bulletin of American Mathematical Society ", (1939).

(2) Slichter L. B., The interpretation of the Resistivity Prospecting Method for Horizontal Structures. "Physics ", Sept. 1933.

( $\left.{ }^{3}\right)$ Chetaev A. L., On the solution of the inverse problem of the theory of electromagnetic soundings. Ac. Sc. U.R.S.S. 1959, "Izvestiya ". 12, (1958).

(4) BeLluigi A', Berechnung der elektrischen Leitfähigkeit des Bodens bei bekannter Verteilung des oberfä̈chtenpotentials. "Gerlands Beiträge Geoph * 3, (1965).

(5) Belluigi A., Die Methode Stevenson zur Ermittlung der eleltrischen Leitfähigkeit aus der Potentialverteilung auf der Begrenzungsebene eines Halbraumes. "Gerlands Beitrüge zur Geoph ", 1956.

${ }^{(}{ }^{8}$ Belluigi A., Über ein geoelektrischen inverses Problem. "Zeit. für Geophysik », 1956.

(') Belluigi A., Neue Theorie für elektrische Sondierungen. "Zeit für Geophysik ", H, 3, 1959. 